\title{
A New Default Probability Calculation Formula and Its Application under Uncertain Environments
}

\author{
Liang Wu $(\mathbb{D})^{1,2}$ Xian-bin Mei, ${ }^{1}$ and Jian-guo Sun $\mathbb{D}^{2}$ \\ ${ }^{1}$ Postdoctoral Research Base, Henan Institute of Science and Technology, Xinxiang 453003, Henan, China \\ ${ }^{2}$ Postdoctoral Research Station, Henan University, Kaifeng 475000, China \\ Correspondence should be addressed to Liang Wu; nidewuliang@163.com
}

Received 20 March 2018; Revised 31 May 2018; Accepted 10 June 2018; Published 1 August 2018

Academic Editor: Beatrice Di Bella

Copyright (c) 2018 Liang Wu et al. This is an open access article distributed under the Creative Commons Attribution License, which permits unrestricted use, distribution, and reproduction in any medium, provided the original work is properly cited.

\begin{abstract}
In the real world, corporate defaults will be affected by both external market shocks and counterparty risks. With this in mind, we propose a new default intensity model with counterparty risks based on both external shocks and the internal contagion effect. The effects of the external shocks and internal contagion on a company cannot, however, be observed, as uncertainty in the real world contains both randomness and fuzziness. This prevents us from determining the size of the shocks accurately. In this study, fuzzy set theory is utilized to study a looping default credit default swap (CDS) pricing model under uncertain environments. Following this, we develop a new fuzzy form pricing formula for CDS, the simulation analysis of which shows that all kinds of fuzziness in the market have a significant impact on credit spreads, and that the credit spreads, relative to the degree of external shock fuzziness, are much more sensitive. Nevertheless, for a certain degree of fuzziness in the market, credit spreads, relative to changes in counterparty risk, are much more sensitive. Using random analysis and fuzzy numbers, one can think of even more uncertain sources at play than the processes of looping default and investor subjective judgment on the financial markets, and this broadens the scope of possible credit spreads. Compared to the existing related literature, our new fuzzy form CDS pricing model with counterparty risk can consider more factors that influence default and is closer to the reality of the complexity of the dynamics of default. It can also employ the membership function to describe the fuzzy phenomenon, enable the fuzzy phenomenon to be estimated in two kinds of state, and can simultaneously reflect both the fuzziness and randomness in financial markets.
\end{abstract}

\section{Introduction}

Information disclosure plays a role as a bridge of information asymmetry between issuers and investors, for disclosure can reduce information asymmetry. Credit derivatives, as nonstandardized financial derivatives, have the following characteristics: the information of trading environment not open to the public, no guarantee of the performance from stock exchange, high risk of performance, and volatile price floating. As a result, the asymmetry of market information flow that acts as a decisive factor of the sensitive credit risk pricing of financial instruments appears. Moreover, it will lead to obvious fuzziness in the process of characterization of the counterparty risk. For example, when some emergencies are caused by financial institution as stock and stock markets, the adverse information dissemination will lead to counterparty default behavior. Meanwhile, the internal market credit default will be convergent and infectious. The counterparty default is featured by synchronicity, aggregation, fuzziness, and uncertainty. In the credit default swap pricing of counterparty risk, the external market shocks and internal contagion effects on the company's size cannot be observed; i.e., the precise value cannot be gotten through a set of random values. Similarly, in the process of risk analysis and other derivatives pricing, people will encounter many similar fuzziness due to the lack of cognition on financial markets, which leads to incapability of seizing market information. For instance, in factor copulas model, correlation cannot be observed between the company's assets. That is to say, people will face the ubiquity of fuzziness in the process of financial risk analysis and its derivatives pricing. As a consequence, the study of fuzziness, hesitation, presentation, and evolution under the condition of asymmetric information is extremely important to control the outbreak, diffusion, and aggregation of the 
credit risk. In addition, the pricing model by combining fuzziness and randomness under the condition of asymmetric information can strip, transfer, and hedge credit risk more efficiently. Thus, the credit risk analysis in the fuzzy uncertain environment and derivatives pricing model in this paper is of great realistic and theoretical significance.

As pointed out in [1-5], in the real world, corporate defaults will be affected by both external market shocks and counterparty risks. With this in mind, we proposed a new default intensity model with counterparty risks based on both external shocks and the internal contagion effect. However, the effects of external shocks and internal contagion on a company cannot be observed, because uncertainty in the real world contains randomness and fuzziness [6-13]. This prevents us from determining the size of shocks accurately. Therefore, inspired by [14-18], fuzzy set theory is adopted to study a looping default credit default swap (CDS) pricing model under uncertain environments. Following this, we set up a new fuzzy form pricing formula for CDS, the simulation analysis of which shows that all kinds of fuzziness in the market have a significant impact on credit spreads. Using random analysis and fuzzy numbers, one can think of more uncertain available sources than the processes of looping default and investor subjective judgment on the financial markets, which broadens the scope of possible credit spreads. Compared to the existing related references [19-23], our new fuzzy form CDS pricing model with counterparty risk can consider more factors that influence default and is closer to the reality of complex default dynamics. It can also employ the membership function to describe the fuzzy phenomenon, enable the fuzzy phenomenon to be estimated in two kinds of state, and simultaneously reflect the fuzziness and randomness in financial markets.

\section{Introducing the Fuzziness to the CDS Pricing Model with Counterparty Risk}

In this section, we study the CDS pricing model under fuzzy random environments based on the new looping default model, and the basic concept of fuzzy set theory can refer to literature [24-27].

2.1. The New Looping Default Intensity Model with Counterparty Risk. Firstly, we introduce some basic concepts. Let $\left(\Omega, F,\left(F_{t}\right)_{0 \leq t \leq T^{*}}, Q\right)$ be a complete probability space. Here, $\left(F_{t}\right)_{0 \leq t \leq T^{*}}$ is a càdlàg, $F=F_{T^{*}}, T^{*}$ is the time limit for uncertain economic system, and $Q$ is the equivalent martingale measure. Suppose that there are $i=1.2, \cdots, n$ companies in the market, and stochastic default time of each company is expressed as $\tau^{i}(i=1,2, \cdots, n)$; the external shock arrival time is expressed as $\tau^{E}$. According to [1], we define the default time as $\tau^{i}:=\inf \left\{t \geq 0: \int_{0}^{t} \lambda_{s}^{i} d s \geq E_{i}\right\}$, where $E_{i}$ is a unit exponential random variable. Meanwhile, the external shock arrival time is assumed to obey uniform distribution in the time interval $[0, T]$. Let $F_{t}=\sigma\left(1_{\left\{\tau^{E} \leq s\right\}}, 0 \leq\right.$ $s \leq t) \vee \sigma\left(1_{\left\{\tau^{1} \leq s\right\}}, 0 \leq s \leq t\right) \vee \cdots \vee \sigma\left(1_{\left\{\tau^{n} \leq s\right\}}, 0 \leq s \leq t\right)$ represent the information sets. So, the conditional survival probability of company i can be expressed as $F^{i}(t)=P\left(\tau^{i} \geq t \mid F_{t}\right)=$ $\exp \left(-\int_{0}^{t} \lambda_{s}^{i} d s\right)$.

For considering more factors that influence default and that are closer to the reality of the complexity of the dynamics of default, we propose a new model as follows:

$$
\lambda_{t}^{i}=a_{0}^{i}+a_{0}^{i}\left(a_{1}^{i}-1\right) 1_{\left\{\tau^{E} \leq t\right\}}+\sum_{k=1, k \neq i}^{n} a^{i, k} 1_{\left\{\tau^{k} \leq t\right\}}
$$

where $a_{0}^{i}>0, a_{1}^{i}>1$, and $a^{i, k}$ represent the initial default intensity, the upward jump ratio, and the counterparty risk, respectively, and $1_{\left\{\tau^{i} \leq t\right\}}$ is an indicator function of company i; i.e., if the company i defaults, then the function value is 1 ; otherwise it is 0 .

To simplify the discussion, we discuss the following:

$$
\begin{aligned}
& \lambda_{t}^{B}=b_{0}+b_{0}\left(b_{1}-1\right) 1_{\left\{\tau^{E} \leq t\right\}}+b_{2} 1_{\left\{\tau^{c} \leq t\right\}} \\
& \lambda_{t}^{c}=c_{0}+c_{0}\left(c_{1}-1\right) 1_{\left\{\tau^{E} \leq t\right\}}+c_{2} 1_{\left\{\tau^{B} \leq t\right\}}
\end{aligned}
$$

Without loss of generality, we assume that before moment $\mathrm{t}$, shock events happen, so (2) and (3) can be written as

$$
\begin{aligned}
& \lambda_{t}^{B}=b_{0} b_{1}+b_{2} 1_{\left\{\tau^{c} \leq t\right\}} \\
& \lambda_{t}^{c}=c_{0} c_{1}+c_{2} 1_{\left\{\tau^{B} \leq t\right\}}
\end{aligned}
$$

In order to begin the operation in the new measure $P^{i}$, we have the following lemmas.

Lemma 1 ([28]). Suppose the parameter $\lambda$ is the intensity process of default time relative to the probability space $(F, P)$; if there exist a constant $p \in[1, \infty)$ and a nonrandom time $\theta$, which meets the integrability condition, $E\left[\exp \left(\mathrm{p} \int_{0}^{\theta} \lambda_{\mu} d \mu\right)\right]<$ $\infty$; at the same time, we set a nonrandom time $T \leq \theta$; then the nonnegative process

$$
Z_{t}:=1_{\{\tau>\mathrm{t} \wedge \mathrm{T}\}} \exp \left(\int_{0}^{\mathrm{t} \wedge \mathrm{T}} \lambda_{\mu} d \mu\right)
$$

is a uniformly integrable martingale relative to the probability space $(F, P)$, and the process is almost always positive in the time interval $[0, \tau)$.

Lemma 2 ([29]). Suppose there exists a probability space $\left(\Omega, G,\left\{G_{t}\right\}_{t \geq 0}, P\right)$, and $Q$ is a new absolutely continuous probability measure relative to the measure $P,\left\{Z_{t}\right\}_{t \geq 0}$ is the derivation process of the corresponding Radon-Nikodym, and if $X$ is $\mathrm{G}_{t}$-measurable, then we have the following conclusion: $E^{\mathrm{Q}}[X]=E^{P}\left[X \cdot Z_{t}\right]$.

Theorem 3. The joint survival probability of different companies and the marginal survival probability of a single company for formulas (4) and (5), are, respectively, as follows: if $0 \leq t_{2} \leq$ $t_{1} \leq T^{*}$, we have the following conclusion: 
Discrete Dynamics in Nature and Society

3

$$
P\left(\tau^{B}>t_{1}, \tau^{C}>t_{2}\right)= \begin{cases}\frac{c_{0} c_{1}}{\left(b_{2}-c_{0} c_{1}\right)} \mathrm{e}^{-\left(b_{0} b_{1}+b_{2}\right) t_{1}}\left[\mathrm{e}^{\left(b_{2}-c_{0} c_{1}\right) t_{1}}-\mathrm{e}^{\left(b_{2}-c_{0} c_{1}\right) t_{2}}\right]+\mathrm{e}^{-\left(c_{0} c_{1}+b_{0} b_{1}\right) t_{1}}, & \mathrm{~b}_{2} \neq c_{0} c_{1} \\ \mathcal{c}_{0} c_{1} \mathrm{e}^{-\left(b_{0} b_{1}+b_{2}\right) t_{1}}\left[t_{1}-t_{2}\right]+\mathrm{e}^{-\left(c_{0} c_{1}+b_{0} b_{1}\right) t_{1}}, & \mathrm{~b}_{2}=c_{0} c_{1}\end{cases}
$$

and if $0 \leq t_{1} \leq t_{2} \leq T^{*}$, we have

$$
P\left(\tau^{B}>t_{1}, \tau^{C}>t_{2}\right)= \begin{cases}\frac{b_{0} b_{1}}{\left(c_{2}-b_{0} b_{1}\right)} \mathrm{e}^{-\left(c_{0} c_{1}+c_{2}\right) t_{2}}\left[\mathrm{e}^{\left(c_{2}-b_{0} b_{1}\right) t_{2}}-\mathrm{e}^{\left(c_{2}-b_{0} b_{1}\right) t_{1}}\right]+\mathrm{e}^{-\left(c_{0} c_{1}+b_{0} b_{1}\right) t_{2}}, & c_{2} \neq b_{0} b_{1} \\ b_{0} b_{1} \mathrm{e}^{-\left(c_{0} c_{1}+c_{2}\right) t_{2}}\left[t_{2}-\mathrm{t}_{1}\right]+\mathrm{e}^{-\left(c_{0} c_{1}+b_{0} b_{1}\right) t_{2}}, & c_{2}=b_{0} b_{1}\end{cases}
$$

The single company's marginal survival probability is as follows: if $0 \leq t_{2} \leq t_{1} \leq T^{*}$, we have

$$
\begin{aligned}
& P\left(\tau^{B}>t\right) \\
& = \begin{cases}\frac{c_{0} c_{1}}{\left(b_{2}-c_{0} c_{1}\right)} \mathrm{e}^{-\left(b_{0} b_{1}+b_{2}\right) t}\left[\mathrm{e}^{\left(b_{2}-c_{0} c_{1}\right) t}-1\right]+\mathrm{e}^{-\left(c_{0} c_{1}+b_{0} b_{1}\right) t}, & \mathrm{~b}_{2} \neq c_{0} c_{1} \\
c_{0} c_{1} \mathrm{e}^{-\left(b_{0} b_{1}+b_{2}\right) t} t+\mathrm{e}^{-\left(c_{0} c_{1}+b_{0} b_{1}\right) t}, & \mathrm{~b}_{2}=c_{0} c_{1}\end{cases}
\end{aligned}
$$

and if $0 \leq t_{1} \leq t_{2} \leq T^{*}$, we have

$$
\begin{aligned}
& P\left(\tau^{C}>t\right) \\
& = \begin{cases}\frac{b_{0} b_{1}}{\left(c_{2}-b_{0} b_{1}\right)} \mathrm{e}^{-\left(c_{0} c_{1}+c_{2}\right) t}\left[\mathrm{e}^{\left(c_{2}-b_{0} b_{1}\right) t}-1\right]+\mathrm{e}^{-\left(c_{0} c_{1}+b_{0} b_{1}\right) t}, & c_{2} \neq b_{0} b_{1} \\
b_{0} b_{1} \mathrm{e}^{-\left(c_{0} c_{1}+c_{2}\right) t} t+\mathrm{e}^{-\left(c_{0} c_{1}+b_{0} b_{1}\right) t}, & c_{2}=b_{0} b_{1}\end{cases}
\end{aligned}
$$

Proof. By Lemmas 1 and 2, we have the following: if $0 \leq t_{2} \leq$ $t_{1} \leq T^{*}$, where $\left(\tau^{B}>t_{1}, \tau^{C}>t_{2}\right) \in F_{t_{1}}$ and $b_{2} \neq c_{0} c_{1}$, then we can derive the following:

$$
\begin{aligned}
P( & \left.\tau^{B}>t_{1}, \tau^{C}>t_{2}\right) \\
= & \mathrm{E}^{B}\left[1_{\left\{\tau^{C}>t_{2}\right\}} \exp \left\{-\int_{0}^{t_{1}}\left(b_{0} b_{1}+b_{2} 1_{\left\{\tau^{C} \leq t\right\}}\right) d t\right\}\right] \\
= & \mathrm{E}^{B}\left[1_{\left\{\tau^{C}>t_{2}\right\}} \mathrm{e}^{-b_{0} b_{1} t_{1}} \exp \left\{-1_{\left\{\tau^{C} \leq t_{1}\right\}} b_{2}\left(\mathrm{t}_{1}-\tau^{C}\right)\right\}\right] \\
= & \mathrm{e}^{-b_{0} b_{1} t_{1}} \mathrm{E}^{B}\left[1_{\left\{t_{2}<\tau^{C} \leq t_{1}\right\}} \mathrm{e}^{-b_{2}\left(\mathrm{t}_{1}-\tau^{C}\right)}+1_{\left\{\tau^{C}>t_{1}\right\}}\right] \\
= & \mathrm{e}^{-b_{0} b_{1} t_{1}}\left[\int_{t_{2}}^{t_{1}} c_{0} c_{1} \mathrm{e}^{-c_{0} c_{1} t} \mathrm{e}^{-b_{2}\left(\mathrm{t}_{1}-t\right)} d t+\mathrm{e}^{-c_{0} c_{1} t_{1}}\right] \\
= & \frac{c_{0} c_{1}}{\left(b_{2}-c_{0} c_{1}\right)} \mathrm{e}^{-\left(b_{0} b_{1}+b_{2}\right) t_{1}}\left[\mathrm{e}^{\left(b_{2}-c_{0} c_{1}\right) t_{1}}-\mathrm{e}^{\left(b_{2}-c_{0} c_{1}\right) t_{2}}\right] \\
& +\mathrm{e}^{-\left(c_{0} c_{1}+b_{0} b_{1}\right) t_{1}},
\end{aligned}
$$

$$
\begin{aligned}
P & \left(\tau^{B}>t_{1}, \tau^{C}>t_{2}\right) \\
& =\mathrm{E}^{B}\left[1_{\left\{\tau^{C}>t_{2}\right\}} \exp \left\{-\int_{0}^{t_{1}}\left(b_{0} b_{1}+b_{2} 1_{\left\{\tau^{C} \leq t\right\}}\right) d t\right\}\right] \\
& =\mathrm{E}^{B}\left[1_{\left\{\tau^{C}>t_{2}\right\}} \mathrm{e}^{-b_{0} b_{1} t_{1}} \exp \left\{-1_{\left\{\tau^{C} \leq t_{1}\right\}} b_{2}\left(\mathrm{t}_{1}-\tau^{C}\right)\right\}\right] \\
& =\mathrm{e}^{-b_{0} b_{1} t_{1}} \mathrm{E}^{B}\left[1_{\left\{t_{2}<\tau^{C} \leq t_{1}\right\}} \mathrm{e}^{-b_{2}\left(\mathrm{t}_{1}-\tau^{C}\right)}+1_{\left\{\tau^{C}>t_{1}\right\}}\right] \\
& =\mathrm{e}^{-b_{0} b_{1} t_{1}}\left[c_{0} c_{1} \mathrm{e}^{-b_{2} t_{1}} \int_{t_{2}}^{t_{1}} \mathrm{e}^{\left(b_{2}-c_{0} c_{1}\right) t} d t+\mathrm{e}^{-c_{0} c_{1} t_{1}}\right] \\
& =c_{0} c_{1} \mathrm{e}^{-\left(b_{0} b_{1}+b_{2}\right) t_{1}}\left[t_{1}-t_{2}\right]+\mathrm{e}^{-\left(c_{0} c_{1}+b_{0} b_{1}\right) t_{1}},
\end{aligned}
$$

if $0 \leq t_{1} \leq t_{2} \leq T^{*}$, where $\left(\tau^{B}>t_{1}, \tau^{C}>t_{2}\right) \in F_{t_{2}}$ and $c_{2} \neq b_{0} b_{1}$, then there are

$$
\begin{aligned}
P( & \left.\tau^{B}>t_{1}, \tau^{C}>t_{2}\right) \\
= & \mathrm{E}^{C}\left[1_{\left\{\tau^{B}>t_{1}\right\}} \exp \left\{-\int_{0}^{t_{2}}\left(c_{0} c_{1}+c_{2} 1_{\left\{\tau^{B} \leq t\right\}}\right) d t\right\}\right] \\
= & \mathrm{E}^{C}\left[1_{\left\{\tau^{B}>t_{1}\right\}} \mathrm{e}^{-c_{0} c_{1} t_{2}} \exp \left\{-1_{\left\{\tau^{B} \leq t_{2}\right\}} c_{2}\left(\mathrm{t}_{2}-\tau^{B}\right)\right\}\right] \\
= & \mathrm{e}^{-c_{0} c_{1} t_{2}} \mathrm{E}^{C}\left[1_{\left\{t_{1}<\tau^{B} \leq t_{2}\right\}} \mathrm{e}^{-c_{2}\left(\mathrm{t}_{2}-\tau^{B}\right)}+1_{\left\{\tau^{B}>t_{2}\right\}}\right] \\
= & \mathrm{e}^{-c_{0} c_{1} t_{2}}\left[\int_{t_{1}}^{t_{2}} b_{0} b_{1} \mathrm{e}^{-b_{0} b_{1} t} \mathrm{e}^{-c_{2}\left(\mathrm{t}_{2}-t\right)} d t+\mathrm{e}^{-b_{0} b_{1} t_{2}}\right] \\
= & \frac{b_{0} b_{1}}{\left(c_{2}-b_{0} b_{1}\right)} \mathrm{e}^{-\left(c_{0} c_{1}+c_{2}\right) t_{2}}\left[\mathrm{e}^{\left(c_{2}-b_{0} b_{1}\right) t_{2}}-\mathrm{e}^{\left(c_{2}-b_{0} b_{1}\right) t_{1}}\right] \\
& +\mathrm{e}^{-\left(c_{0} c_{1}+b_{0} b_{1}\right) t_{2}},
\end{aligned}
$$


and if $c_{2}=b_{0} b_{1}$, we can derive the following:

$$
\begin{aligned}
P & \left(\tau^{B}>t_{1}, \tau^{C}>t_{2}\right) \\
& =\mathrm{E}^{C}\left[1_{\left\{\tau^{B}>t_{1}\right\}} \exp \left\{-\int_{0}^{t_{2}}\left(c_{0} c_{1}+c_{2} 1_{\left\{\tau^{B} \leq t\right\}}\right) d t\right\}\right] \\
& =\mathrm{E}^{C}\left[1_{\left\{\tau^{B}>t_{1}\right\}} \mathrm{e}^{-c_{0} c_{1} t_{2}} \exp \left\{-1_{\left\{\tau^{B} \leq t_{2}\right\}} c_{2}\left(\mathrm{t}_{2}-\tau^{B}\right)\right\}\right] \\
& =\mathrm{e}^{-c_{0} c_{1} t_{2}} \mathrm{E}^{C}\left[1_{\left\{t_{1}<\tau^{B} \leq t_{2}\right\}} \mathrm{e}^{-c_{2}\left(\mathrm{t}_{2}-\tau^{B}\right)}+1_{\left\{\tau^{B}>t_{2}\right\}}\right] \\
& =\mathrm{e}^{-c_{0} c_{1} t_{2}}\left[b_{0} b_{1} \mathrm{e}^{-c_{2} t_{2}} \int_{t_{1}}^{t_{2}} \mathrm{e}^{\left(c_{2}-b_{0} b_{1}\right) t} d t+\mathrm{e}^{-b_{0} b_{1} t_{2}}\right] \\
& =b_{0} b_{1} \mathrm{e}^{-\left(c_{0} c_{1}+c_{2}\right) t_{2}}\left[t_{2}-\mathrm{t}_{1}\right]+\mathrm{e}^{-\left(c_{0} c_{1}+b_{0} b_{1}\right) t_{2}} .
\end{aligned}
$$

The proof of the marginal survival probability of a single company only needs to take the value $t_{2}=0, t_{1}=0$ in formulas (7) and (8), which completes the proof.

2.2. The CDS Pricing Model with Counterparty Risk under Uncertain Environments. Financial data may not be timely or accurately recorded, due to unforeseen circumstances or man-made errors. In order to reflect the influence of fuzziness on the interest rate, we assume that the interest rate is a triangular fuzzy random variable $\widetilde{r}(t)$,

$$
\mu_{\widetilde{r}_{t}}(x)= \begin{cases}1-\frac{r-x}{\alpha^{-}(t)}, & r-\alpha^{-}(t) \leq x \leq r \\ 1-\frac{x-r}{\alpha^{+}(t)}, & r \leq x \leq r+\alpha^{+}(t) \\ 0, & \text { otherwise }\end{cases}
$$

That is, the triangular fuzzy random variable $\widetilde{r}(t)$ is represented by the center $r$, left-width $\alpha^{-}(t)$, and right-width $\alpha^{+}(t)$ (also known as fuzzy degree factor); see Figure 1. We can find that the fuzziness of investors on the market interest rate in the process increases as $\alpha^{ \pm}(t)$ becomes larger. The $\gamma$-cut of $\widetilde{r}(t)$ is

$$
\begin{aligned}
\tilde{r}_{\gamma}(t) & =\left\{x \mid \mu_{\widetilde{r}_{t}}(x) \geq \gamma\right\} \\
& =\left[r-(1-\gamma) \alpha_{t}^{-}, r+(1-\gamma) \alpha_{t}^{+}\right], \quad \gamma \in[0,1] .
\end{aligned}
$$

Meanwhile, the influences of external market shocks $b_{1}, c_{1}$ and counterparty risks $b_{2}, c_{2}$ are assumed to be triangular fuzzy numbers, whose membership functions are defined as follows:

$$
\begin{aligned}
& b_{1}(x)=\max \left\{1-\frac{\left|x-b_{1}\right|}{\beta_{1}}, 0\right\}, \\
& c_{1}(x)=\max \left\{1-\frac{\left|x-c_{1}\right|}{\beta_{2}}, 0\right\}, \\
& b_{2}(x)=\max \left\{1-\frac{\left|x-b_{2}\right|}{\kappa_{1}}, 0\right\}, \\
& c_{2}(x)=\max \left\{1-\frac{\left|x-c_{2}\right|}{\kappa_{2}}, 0\right\},
\end{aligned}
$$

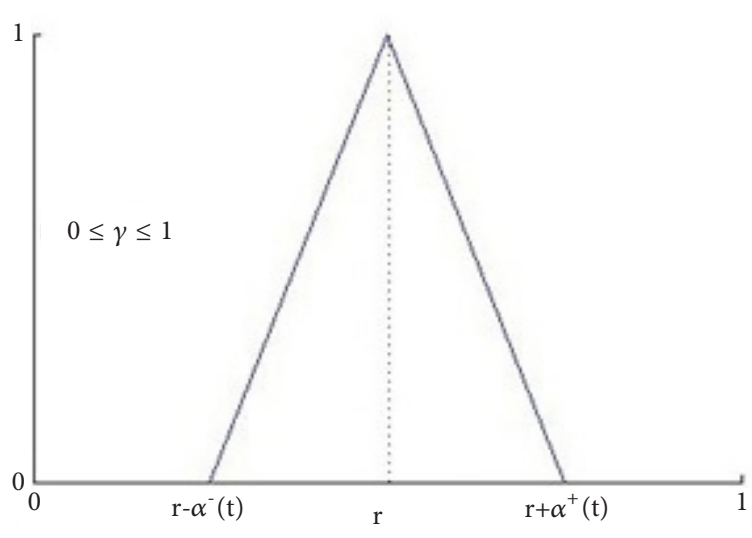

FiguRE 1: The triangular fuzzy risk-free interest rate.

that is, the fuzzy numbers $\widetilde{b}_{1}, \widetilde{c}_{1}$ and $\widetilde{b}_{2}, \widetilde{c}_{2}$ have a symmetric triangle-type shape, respectively, with centers $b_{1}, c_{1}$ and $b_{2}$, $c_{2}$, and fuzzy degree factors $\beta_{1}, \beta_{2}$ and $\kappa_{1}, \kappa_{2}$. The rationale behind fuzzy external market shocks and counterparty risk lies in the difficulty of getting a precise estimate of the actual external shock and counterparty risk of default intensity. By modeling the external shocks and counterparty risk as a fuzzy number, one can take the investors' subjective judgment into account.

According to [7], we introduce a reasonable assumption as follows to simplify the discussion.

Assumption 4. The stochastic process $\alpha^{ \pm}(t)$ is specified by $\alpha^{ \pm}(t)=c^{ \pm} r$, where $0<c^{-}, c^{+}<1$. Similarly, the values $\beta_{1}$, $\beta_{2}$ are specified by $\beta_{1}=a b_{1}, \beta_{2}=a c_{1}$, where $0<a<1$; the values $\kappa_{1}, \kappa_{2}$ are specified by $\kappa_{1}=b b_{2}, \kappa_{2}=b c_{2}$, where $0<b<1$.

Assumption 4 is reasonable since $\alpha^{ \pm}(t)$ and $\beta_{1}, \beta_{2}$ and $\kappa_{1}$, $\kappa_{2}$ are related to the fuzziness of the volatility in the financial environment. Thus,

$$
\begin{aligned}
& \tilde{r}_{t, \gamma}=\left[\left(1-(1-\gamma) c^{-}\right) r,\left(1+(1-\gamma) c^{+}\right) r\right], \\
& \tilde{b}_{1, \gamma}=\left[(1-(1-\gamma) a) b_{1},(1+(1-\gamma) a) b_{1}\right], \\
& \tilde{c}_{1, \gamma}=\left[(1-(1-\gamma) a) c_{1},(1+(1-\gamma) a) c_{1}\right], \\
& \tilde{b}_{2, \gamma}=\left[(1-(1-\gamma) b) b_{2},(1+(1-\gamma) b) b_{2}\right], \\
& \tilde{c}_{2, \gamma}=\left[(1-(1-\gamma) b) c_{2},(1+(1-\gamma) b) c_{2}\right], \quad \gamma \in[0,1] .
\end{aligned}
$$

For CDS pricing, we provide some propositions. Hypothesize that the market short-term interest rate $\tilde{r}(t)$ satisfies the CIR (Cox-Ingersoll-Ross) model; that is,

$$
d \widetilde{r}_{t}=\alpha\left(k-\widetilde{r}_{t}\right) d t+\sigma \sqrt{\widetilde{r}_{t}} d W_{t}
$$


Therefore, the default-free zero coupon bond price, whose the face value is $\$ 1$ and expiration date is $T<T^{\circ}$, can be calculated by the following formula:

$$
\widetilde{p}(t, T)=E^{Q}\left[\exp \left(-\int_{t}^{T} \tilde{r}_{s} d s\right)\right] .
$$

According to Theorem 4.3.1 of [30], the conclusions are $\widetilde{p}(t, T)=e^{-\widetilde{r}_{t} C(t, T)-A(t, T)}$,

$$
C(t, T)
$$

$$
\begin{aligned}
& =\frac{e^{\rho(T-t)}-e^{-\rho(T-t)}}{(\rho+(1 / 2) \alpha) e^{\rho(T-t)}+(\rho-(1 / 2) \alpha) e^{-\rho(T-t)}}, \\
A(t, T)=-\alpha k \int_{t}^{T} C(s, T) d s, & \\
\rho & =\frac{1}{2} \sqrt{\alpha^{2}+2 \sigma^{2}} .
\end{aligned}
$$

Theorem 5. Suppose that the market short-term interest rate $\widetilde{r}(t)$ satisfies the CIR model, then, the $\gamma$-cut of the price of default-free zero coupon bond, whose face value is $\$ 1$ and expiration date is $T$, can be written as $\tilde{p}_{\gamma}(t, T)=\left[\tilde{p}_{\gamma}^{-}, \tilde{p}_{\gamma}^{+}\right]$, where the left and right ends, respectively, are

$$
\begin{aligned}
& \widetilde{p}_{\gamma}^{-}(t, T)=e^{-\left(1+(1-\gamma) c^{+}\right) r C(t, T)-A(t, T)}, \\
& \widetilde{p}_{\gamma}^{+}(t, T)=e^{-\left(1-(1-\gamma) c^{-}\right) r C(t, T)-A(t, T)}, \\
& \gamma \in[0,1] .
\end{aligned}
$$

Proof. According to the monotonicity of the function and fuzzy number operation rule, we have the conclusion.

At moment $t$, the price of defaultable zero coupon bonds is equal to the discounted future cash flow:

$$
\begin{array}{r}
\widetilde{V}^{i}(t, T) \\
=E^{Q}\left[\exp \left(-\int_{t}^{T} \widetilde{r}_{\mu} d \mu\right)\left(\beta^{i} 1_{\left\{\tau^{i} \leq T\right\}}+1_{\left\{\tau^{i}>T\right\}}\right)\right] \\
(i=B, C),
\end{array}
$$

where $\beta^{i}$ is the default recovery rate of $i$ company; to simplify the analysis, the following discussion assumes that $\beta^{i}=0$.

Now, we develop a CDS pricing model under uncertain environments based on the new looping default models (4) and (5).

The present fuzzy value of fixed leg is

$P V($ fixed leg)

$$
=E^{Q}\left[\widetilde{s} \int_{0}^{T} \exp \left(-\int_{0}^{t} \widetilde{r}_{\mu} d \mu\right) 1_{\left\{\tau^{B}>t, \tau^{C}>t\right\}} d t \mid H_{t}\right] .
$$

The present fuzzy value of default leg is

$$
\begin{aligned}
& P V(\text { default leg }) \\
& \quad=E^{\mathrm{Q}}\left[1_{\left\{\tau^{\mathrm{C}} \leq T\right\}} \exp \left(-\int_{0}^{T} \widetilde{r}_{\mu} d \mu\right) 1_{\left\{\tau^{B}>T\right\}} \mid H_{t}\right] .
\end{aligned}
$$

Theorem 6. The present fuzzy value of a credit default swap $\widetilde{\boldsymbol{s}}$ is given as

$$
\widetilde{s}=\frac{\widetilde{V}^{B}(0, T)-e^{-b_{0} \widetilde{b}_{1} T} \widetilde{V}^{C}(0, T)}{\int_{0}^{T} \widetilde{p}(0, \mu) e^{-\left(b_{0} \widetilde{b}_{1}+c_{0} \widetilde{c}_{1}\right) \mu} d \mu}
$$

The $\gamma$-cut of $\widetilde{s}$ is calculated as $\widetilde{s}_{\gamma}=\left[\widetilde{s}_{\gamma}, \widetilde{s}_{\gamma}^{+}\right]$, where the left and right endpoints are

$$
\begin{aligned}
& \widetilde{s}_{\gamma}=\frac{\widetilde{V}_{\gamma}^{B,-}(0, T)-e^{-b_{0}(1-(1-\gamma) a) b_{1} T} \widetilde{V}_{\gamma}^{C,+}(0, T)}{\int_{0}^{T} \widetilde{p}_{\gamma}^{+}(0, \mu) e^{-\left(b_{0}(1-(1-\gamma) a) b_{1}+c_{0}(1-(1-\gamma) a) c_{1}\right) \mu} d \mu}, \\
& \widetilde{s}_{\gamma}^{+}=\frac{\widetilde{V}_{\gamma}^{B,+}(0, T)-e^{-b_{0}(1+(1-\gamma) a) b_{1} T} \widetilde{V}_{\gamma}^{C,-}(0, T)}{\int_{0}^{T} \widetilde{p}_{\gamma}^{-}(0, \mu) e^{-\left(b_{0}(1+(1-\gamma) a) b_{1}+c_{0}(1+(1-\gamma) a) c_{1}\right) \mu} d \mu},
\end{aligned}
$$

and where

$$
\begin{aligned}
& \widetilde{V}_{\gamma}^{B,-}(0, T)= \begin{cases}\tilde{p}_{\gamma}^{-}(0, T)\left\{\frac{c_{0}(1-(1-\gamma) a) c_{1}}{\left((1+(1-\gamma) b) b_{2}-c_{0}(1-(1-\gamma) a) c_{1}\right)} \mathrm{e}^{-\left(b_{0}(1+(1-\gamma) a) b_{1}+(1+(1-\gamma) b) b_{2}\right) \mathrm{T}} \times\right. \\
\left.\left[\mathrm{e}^{\left((1-(1-\gamma) b) b_{2}-c_{0}(1+(1-\gamma) a) c_{1}\right) \mathrm{T}}-1\right]+\mathrm{e}^{-\left(c_{0}(1+(1-\gamma) a) c_{1}+b_{0}(1+(1-\gamma) a) b_{1}\right) \mathrm{T}}\right\}, & \widetilde{\mathrm{b}}_{2} \neq c_{0} \widetilde{c}_{1} \\
\tilde{p}_{\gamma}^{-}(0, T)\left\{c_{0}(1-(1-\gamma) a) c_{1} \mathrm{e}^{-\left(b_{0}(1+(1-\gamma) a) b_{1}+(1+(1-\gamma) b) b_{2}\right) \mathrm{T}} T+\mathrm{e}^{-\left(c_{0}(1+(1-\gamma) a) c_{1}+b_{0}(1+(1-\gamma) a) b_{1}\right) \mathrm{T}}\right\}, & \widetilde{\mathrm{b}}_{2}=c_{0} \widetilde{c}_{1}\end{cases} \\
& \widetilde{V}_{\gamma}^{B,+}(0, T)= \begin{cases}\tilde{p}_{\gamma}^{+}(0, T)\left\{\frac{c_{0}(1+(1-\gamma) a) c_{1}}{\left((1-(1-\gamma) b) b_{2}-c_{0}(1+(1-\gamma) a) c_{1}\right)} \mathrm{e}^{-\left(b_{0}(1-(1-\gamma) a) b_{1}+(1-(1-\gamma) b) b_{2}\right) \mathrm{T}} \times\right. \\
\left.\left[\mathrm{e}^{\left((1+(1-\gamma) b) b_{2}-c_{0}(1-(1-\gamma) a) c_{1}\right) \mathrm{T}}-1\right]+\mathrm{e}^{-\left(c_{0}(1-(1-\gamma) a) c_{1}+b_{0}(1-(1-\gamma) a) b_{1}\right) \mathrm{T}}\right\}, & \widetilde{\mathrm{b}}_{2} \neq c_{0} \widetilde{c}_{1} \\
\tilde{p}_{\gamma}^{+}(0, T)\left\{c_{0}(1+(1-\gamma) a) c_{1} \mathrm{e}^{-\left(b_{0}(1-(1-\gamma) a) b_{1}+(1-(1-\gamma) b) b_{2}\right) \mathrm{T}} T+\mathrm{e}^{-\left(c_{0}(1-(1-\gamma) a) c_{1}+b_{0}(1-(1-\gamma) a) b_{1}\right) \mathrm{T}}\right\}, & \widetilde{\mathrm{b}}_{2}=c_{0} \widetilde{c}_{1}\end{cases}
\end{aligned}
$$




$$
\begin{aligned}
& \widetilde{V}_{\gamma}^{C,-}(0, T)= \begin{cases}\tilde{p}_{\gamma}^{-}(0, T)\left\{\frac{b_{0}(1-(1-\gamma) a) b_{1}}{\left((1+(1-\gamma) b) c_{2}-b_{0}(1-(1-\gamma) a) b_{1}\right)} \mathrm{e}^{-\left(c_{0}(1+(1-\gamma) a) c_{1}+(1+(1-\gamma) b) c_{2}\right) \mathrm{T}} \times\right. \\
\left.\left[\mathrm{e}^{\left((1-(1-\gamma) b) c_{2}-b_{0}(1+(1-\gamma) a) b_{1}\right) \mathrm{T}}-1\right]+\mathrm{e}^{-\left(c_{0}(1+(1-\gamma) a) c_{1}+b_{0}(1+(1-\gamma) a) b_{1}\right) \mathrm{T}}\right\}, \\
\tilde{p}_{\gamma}^{-}(0, T)\left\{b_{0}(1-(1-\gamma) a) b_{1} \mathrm{e}^{-\left(c_{0}(1+(1-\gamma) a) c_{1}+(1+(1-\gamma) b) c_{2}\right) \mathrm{T}} T+\mathrm{e}^{-\left(c_{0}(1+(1-\gamma) a) c_{1}+b_{0}(1+(1-\gamma) a) b_{1}\right) \mathrm{T}}\right\}, & \widetilde{c}_{2}=b_{0} \widetilde{b}_{1}\end{cases} \\
& \widetilde{V}_{\gamma}^{C,+}(0, T)= \begin{cases}\tilde{p}_{\gamma}^{+}(0, T)\left\{\frac{b_{0}(1+(1-\gamma) a) b_{1}}{\left((1-(1-\gamma) b) c_{2}-b_{0}(1+(1-\gamma) a) b_{1}\right)} \mathrm{e}^{-\left(c_{0}(1-(1-\gamma) a) c_{1}+(1-(1-\gamma) b) c_{2}\right) \mathrm{T}} \times\right. \\
\left.\left[\mathrm{e}^{\left((1+(1-\gamma) b) c_{2}-b_{0}(1-(1-\gamma) a) b_{1}\right) \mathrm{T}}-1\right]+\mathrm{e}^{-\left(c_{0}(1-(1-\gamma) a) c_{1}+b_{0}(1-(1-\gamma) a) b_{1}\right) \mathrm{T}}\right\}, \\
\tilde{p}_{\gamma}^{+}(0, T)\left\{b_{0}(1+(1-\gamma) a) b_{1} \mathrm{e}^{-\left(c_{0}(1-(1-\gamma) a) c_{1}+(1-(1-\gamma) b) c_{2}\right) \mathrm{T}} T+\mathrm{e}^{-\left(c_{0}(1-(1-\gamma) a) c_{1}+b_{0}(1-(1-\gamma) a) b_{1}\right) \mathrm{T}}\right\}, & \widetilde{c}_{2}=b_{0} \widetilde{b}_{1}\end{cases}
\end{aligned}
$$

Proof. Based on the no arbitrage principle, as well as the conditional function $P V$ (fixed leg) $=P V$ (default leg), we have the following:

$$
\begin{aligned}
\widetilde{s} & =\frac{E^{\mathrm{Q}}\left[1_{\left\{\tau^{C} \leq T\right\}} \exp \left(-\int_{0}^{T} \tilde{r}_{\mu} d \mu\right) 1_{\left\{\tau^{B}>T\right\}} \mid H_{t}\right]}{E^{\mathrm{Q}}\left[\int_{0}^{T} \exp \left(-\int_{0}^{t} \tilde{r}_{\mu} d \mu\right) 1_{\left\{\tau^{B}>t, \tau^{C}>t\right\}} d t \mid H_{t}\right]} \\
& =\frac{E^{\mathrm{Q}}\left[1_{\left\{\tau^{B}>T\right\}} \exp \left(-\int_{0}^{T} \tilde{r}_{\mu} d \mu\right) \mid H_{t}\right]-E^{\mathrm{Q}}\left[1_{\left\{\tau^{C}>T\right\}} \exp \left(-\int_{0}^{T} \widetilde{r}_{\mu}+\widetilde{\lambda}_{\mu}^{B} d \mu\right) \mid H_{t}\right]}{E^{\mathrm{Q}}\left[\int_{0}^{T} \exp \left(-\int_{0}^{t} \tilde{r}_{\mu} d \mu\right) 1_{\left\{\tau^{B}>t, \tau^{C}>t\right\}} d t \mid H_{t}\right]} \\
& =\frac{\widetilde{V}^{B}(0, T)-E^{\mathrm{Q}}\left[1_{\left\{\tau^{C}>T\right\}} \exp \left(-\int_{0}^{T} \tilde{r}_{\mu}+\widetilde{\lambda}_{\mu}^{B} d \mu\right) \mid H_{t}\right]}{\int_{0}^{T} \tilde{p}(0, \mu) e^{-\left(b_{0} \widetilde{b}_{1}+c_{0} \tilde{c}_{1}\right) \mu} d \mu},
\end{aligned}
$$

where

$$
\begin{aligned}
E^{Q} & {\left[1_{\left\{\tau^{B}>t, \tau^{C}>t\right\}} d t \mid H_{t}\right]=\mathrm{P}\left(\tau^{B}>t, \tau^{C}>t\right) } \\
& =e^{-\left(b_{0} \widetilde{b}_{1}+c_{0} \widetilde{1}_{1}\right) t}, \\
E^{Q} & {\left[1_{\left\{\tau^{C}>T\right\}} \exp \left(-\int_{0}^{T}\left(\widetilde{r}_{\mu}+\tilde{\lambda}_{\mu}^{B}\right) d \mu\right) \mid H_{t}\right] } \\
& =E^{Q}\left[1_{\left\{\tau^{C}>T\right\}}\right. \\
& \left.\cdot \exp \left(-\int_{0}^{T}\left(\widetilde{r}_{\mu}+b_{0} \widetilde{b}_{1}+\widetilde{b}_{2} 1_{\left\{\tau^{C} \leq \mu\right\}}\right) d \mu\right) \mid H_{t}\right] \\
& =E^{Q}\left[1_{\left\{\tau^{C}>T\right\}}\right. \\
& \cdot \exp \left(-b_{0} \widetilde{b}_{1} T-\int_{0}^{T} \widetilde{r}_{\mu} d \mu-\widetilde{b}_{2}\left(T-\tau^{c}\right) 1_{\left\{\tau^{c} \leq T\right\}}\right) \mid
\end{aligned}
$$

$$
\begin{aligned}
& \left.H_{t}\right]=E^{Q}\left[1_{\left\{\tau^{C}>T\right\}} e^{-b_{0} \widetilde{b}_{1} T-\int_{0}^{T} \widetilde{r}_{\mu} d \mu}\right] \\
& =e^{-b_{0} \widetilde{b}_{1} T} E^{Q}\left[1_{\left\{\tau^{C}>T\right\}} e^{-\int_{0}^{T} \widetilde{r}_{\mu} d \mu}\right]=e^{-b_{0} \widetilde{b}_{1} T} \widetilde{V}^{C}(0, T) .
\end{aligned}
$$

Therefore, $\widetilde{s}=\left(\widetilde{V}^{B}(0, T)-e^{-b_{0} \widetilde{b}_{1} T \widetilde{V}^{C}}(0, T)\right) /$ $\int_{0}^{T} \tilde{p}(0, \mu) e^{-\left(b_{0} \widetilde{b}_{1}+c_{0} \widetilde{c}_{1}\right) \mu} d \mu$

Meanwhile, based on the monotony of the functions, we can derive the $\gamma$-cut of $\widetilde{s}_{\gamma}$ as $\widetilde{s}_{\gamma}=\left[\widetilde{s}_{\gamma}, \widetilde{s}_{\gamma}^{+}\right]$, where

$$
\begin{aligned}
\widetilde{s}_{\gamma}^{-} & =\left[\frac{\widetilde{V}^{B}(0, T)-e^{-b_{0} \widetilde{b}_{1} T} \widetilde{V}^{C}(0, T)}{\int_{0}^{T} \widetilde{p}(0, \mu) e^{-\left(b_{0} \widetilde{b}_{1}+c_{0} \widetilde{c}_{1}\right) \mu} d \mu}\right]_{\gamma}^{-} \\
= & \frac{\left[\widetilde{V}^{B}(0, T)\right]_{\gamma}^{-}-\left[e^{-b_{0} \widetilde{b}_{1} T}\right]_{\gamma}^{+}\left[\widetilde{V}^{C}(0, T)\right]_{\gamma}^{+}}{\int_{0}^{T}[\widetilde{p}(0, \mu)]_{\gamma}^{+}\left[e^{-\left(b_{0} \widetilde{b}_{1}+c_{0} \widetilde{c}_{1}\right) \mu}\right]_{\gamma}^{+} d \mu} \\
= & \frac{\widetilde{V}_{\gamma}^{B,-}(0, T)-e^{-b_{0}(1-(1-\gamma) a) b_{1} T} \widetilde{V}_{\gamma}^{C,+}(0, T)}{\int_{0}^{T} \widetilde{p}_{\gamma}^{+}(0, \mu) e^{-\left(b_{0}(1-(1-\gamma) a) b_{1}+c_{0}(1-(1-\gamma) a) c_{1}\right) \mu} d \mu}
\end{aligned}
$$


a similar conclusion $\widetilde{s}_{\gamma}^{+}$can be proved. At the same time, we have

$$
\begin{aligned}
& \widetilde{V}_{\gamma}^{B,-}(0, T)=E^{\mathrm{Q}}\left[\exp \left(-\int_{t}^{T} \widetilde{r}_{\mu} d \mu\right) 1_{\left\{\tau^{B}>T\right\}}\right]_{\gamma}^{-}=[\tilde{p}(0, T)]_{\gamma}^{-} E^{\mathrm{Q}}\left[1_{\left\{\tau^{B}>T\right\}}\right]_{\gamma}^{-}=\widetilde{p}_{\gamma}^{-}(0, T)\left[\mathrm{P}\left(\tau^{B}>\mathrm{T}\right)\right]_{\gamma}^{-} \\
& = \begin{cases}\tilde{p}_{\gamma}^{-}(0, T)\left\{\frac{c_{0} c_{1}}{\left(b_{2-} c_{0} c_{1}\right)} \mathrm{e}^{-\left(b_{0} b_{1}+b_{2}\right) t}\left[\mathrm{e}^{\left(b_{2}-c_{0} c_{1}\right) t}-1\right]+\mathrm{e}^{-\left(c_{0} c_{1}+b_{0} b_{1}\right) t}\right\}_{\gamma}^{-}, & \mathrm{b}_{2} \neq c_{0} c_{1} \\
\tilde{p}_{\gamma}^{-}(0, T)\left\{c_{0} c_{1} \mathrm{e}^{-\left(b_{0} b_{1}+b_{2}\right) t} t+\mathrm{e}^{-\left(c_{0} c_{1}+b_{0} b_{1}\right) t}\right\}_{\gamma}^{-}, & \mathrm{b}_{2}=c_{0} c_{1}\end{cases} \\
& = \begin{cases}\tilde{p}_{\gamma}^{-}(0, T)\left\{\frac{c_{0}(1-(1-\gamma) a) c_{1}}{\left((1+(1-\gamma) b) b_{2}-c_{0}(1-(1-\gamma) a) c_{1}\right)} \mathrm{e}^{-\left(b_{0}(1+(1-\gamma) a) b_{1}+(1+(1-\gamma) b) b_{2}\right) \mathrm{T}} \times\right. \\
\left.\left[\mathrm{e}^{\left((1-(1-\gamma) b) b_{2}-c_{0}(1+(1-\gamma) a) c_{1}\right) \mathrm{T}}-1\right]+\mathrm{e}^{-\left(c_{0}(1+(1-\gamma) a) c_{1}+b_{0}(1+(1-\gamma) a) b_{1}\right) \mathrm{T}}\right\}, & \widetilde{\mathrm{b}}_{2} \neq c_{0} \widetilde{c}_{1} \\
\tilde{p}_{\gamma}^{-}(0, T)\left\{c_{0}(1-(1-\gamma) a) c_{1} \mathrm{e}^{-\left(b_{0}(1+(1-\gamma) a) b_{1}+(1+(1-\gamma) b) b_{2}\right) \mathrm{T}} T+\mathrm{e}^{-\left(c_{0}(1+(1-\gamma) a) c_{1}+b_{0}(1+(1-\gamma) a) b_{1}\right) \mathrm{T}}\right\}, & \widetilde{\mathrm{b}}_{2}=c_{0} \widetilde{c}_{1}\end{cases}
\end{aligned}
$$

where similar conclusions $\widetilde{V}_{\gamma}^{B,+}(0, T), \widetilde{V}_{\gamma}^{C,-}(0, T), \widetilde{V}_{\gamma}^{C,+}(0, T)$ can also be proved, so the proof is completed.

\section{Simulation Analysis}

In this part, we conduct a simulation on Theorem 6 and set the parameter as shown in Table 1.

Other related parameters are considered as follows: in the CIR interest rate model, we suppose that $\alpha=0.03, k=0.04$, and $\sigma=0.07$; in the looping default models (4) and (5), we suppose that the initial default intensity parameters $b_{0}=0.6$, $c_{0}=0.7$ and hypothesize that the triangular fuzzy type riskfree interest rate, external shocks, and counterparty risk are

$$
\begin{aligned}
& \tilde{r}_{t, \gamma}=[(1-(1-\gamma) 0.3) 0.07,(1+(1-\gamma) 0.2) 0.07] \\
& \tilde{b}_{1, \gamma}=[(1-(1-\gamma) 0.1) 1.04,(1+(1-\gamma) 0.1) 1.04] ; \\
& \widetilde{c}_{1, \gamma}=[(1-(1-\gamma) 0.1) 1.07,(1+(1-\gamma) 0.1) 1.07] ; \\
& \widetilde{b}_{2, \gamma}=[(1-(1-\gamma) 0.4) 0.06,(1+(1-\gamma) 0.4) 0.06] ; \\
& \widetilde{c}_{2, \gamma}=[(1-(1-\gamma) 0.4) 0.08,(1+(1-\gamma) 0.4) 0.08] ;
\end{aligned}
$$

where $\gamma \in[0,1]$, which represents the subjective reliability of investors. According to the parameters set by Table 1 and by means of simulation through the use of MATLAB R2010a, we obtain the simulation results in Figures 2-7.

In Figures 2-4, we see that the price range of simulation will become larger as the fuzzy degree of interest rates, external shocks, and counterparty risk increase, with the fuzzy credit spreads related to outside market shocks much more sensitive. This implies that all kinds of fuzziness in the market will have a significant impact on credit spreads. From Figure 5, we can see that, along with the continuous improvement in personal subjective judgment reliability, the fuzzy credit spreads interval is gradually narrowed, until subjective reliability reaches 1 ; at that moment, the price range changes back to a real number; that is, there is no fuzziness in the market. This implies that people can improve their personal subjective judgment reliability to narrow the range of fuzzy credit spreads and select the optimal credit spread range. From Figures 6-7, we can see that external shocks and counterparty risk have a reverse effect on credit spreads (pricing environments with or without fuzziness have the same effect); increasing the external shocks and counterparty risk causes credit spreads to become smaller and smaller. In the fuzzy environment, credit spreads, compared to changes in counterparty risk, are much more sensitive. The above shows that both external shocks and counterparty risk have a significant effect on credit spreads. This is especially true when there is a certain degree of fuzziness in the market, when the spreads are caused by the counterparty risk.

Compared to the literature $[14,17,22,23]$, our new fuzzy form CDS pricing model with counterparty risk has more default influence factors, is much closer to the real complexity of default dynamics, employs the membership function to describe the fuzzy phenomenon, enables the fuzzy phenomenon to be estimated in two kinds of state, the possible degree and impossible degree, and can simultaneously reflect the fuzziness and the randomness in financial markets.

\section{Conclusions}

Using random analysis and fuzzy numbers, one can think of yet more uncertain sources leading to looping default, sources such as the subjective judgment of financial market investors leading to a broadening of possible credit spreads. The proposed model can thus be used as a new tool for CDS pricing. Of course, there are some deficiencies in this paper. Due to limited market data, we did not check market data parameters for the model, and this is what we will study in the future. Likewise, the stability of the model's results is also the focus of our further research. 
TABLE 1: The parameter configuration.

\begin{tabular}{lcc}
\hline $\begin{array}{l}\text { parameter classification } \\
\text { time parameter }\end{array}$ & $\begin{array}{c}\text { parameter name } \\
\text { expiration date } \mathrm{T}\end{array}$ & $\begin{array}{c}\text { parameter configuration } \\
\text { at present, the largest trading volume of CDS contract is } 5 \text { years, therefore, } \\
\text { hypothesize that } \mathrm{T}=5 .\end{array}$ \\
$\begin{array}{c}\text { iscount parameter } \\
\text { default parameter }\end{array}$ & $\begin{array}{c}\text { remploys the period counting rates or yields, suppose that } \mathrm{r}=7 \% . \\
\text { recovery rate } \beta^{i}\end{array}$ & for the convenience of analysis, assume that $\beta^{i}=0$. \\
\hline
\end{tabular}

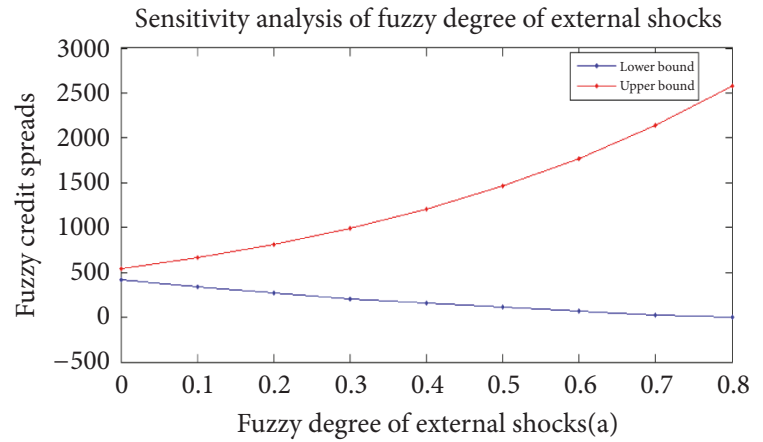

FIGURE 2: The dynamics between fuzzy credit spreads and fuzzy degree of external shocks.

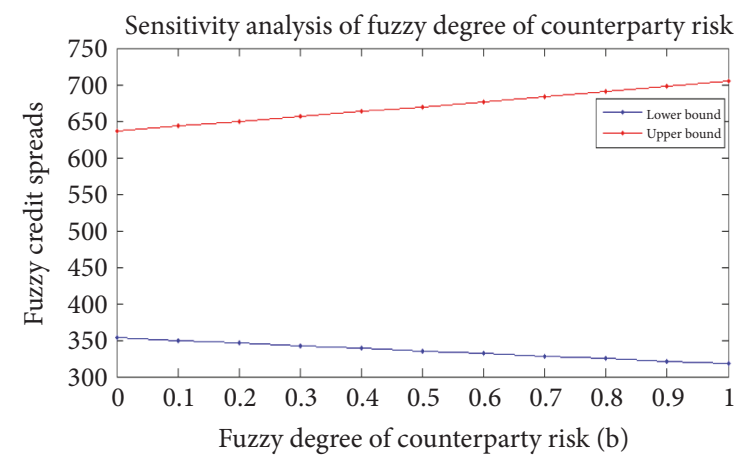

FIgURE 3: The dynamics between fuzzy credit spreads and fuzzy degree of counterparty risk.

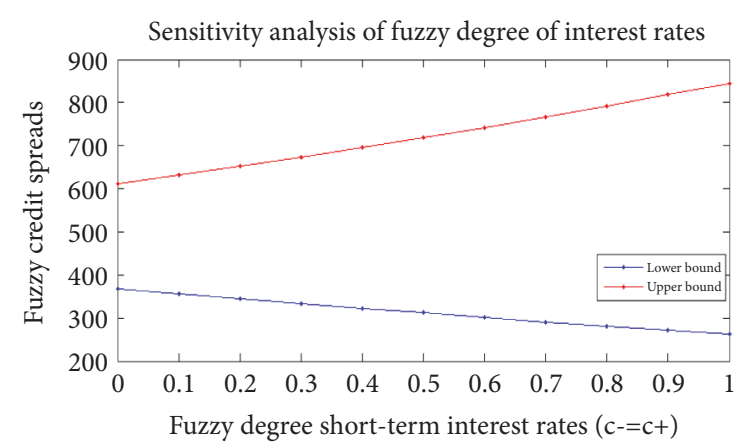

Figure 4: The dynamics between fuzzy credit spreads and fuzzy degree of interest rates.

\section{Data Availability}

The data used to support the findings of this study are available from the corresponding author upon request.

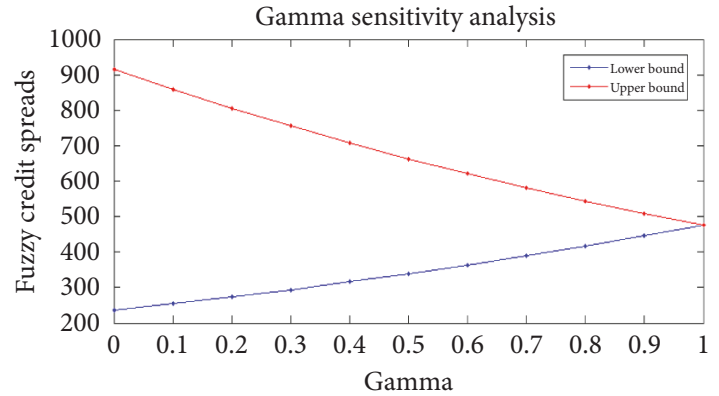

FIGURE 5: The dynamics between fuzzy credit spreads and subjective reliability.

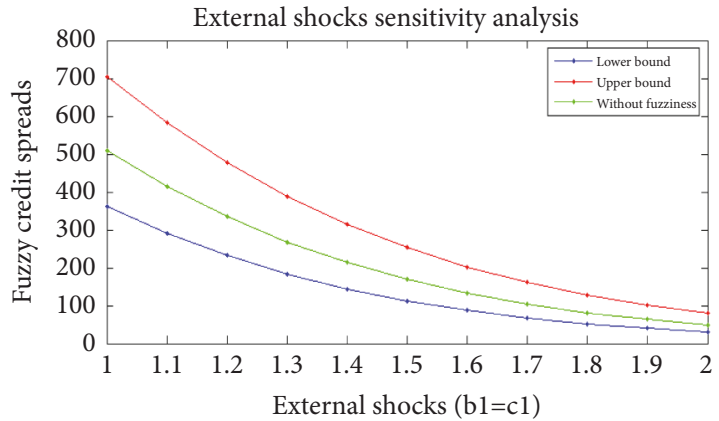

FIgURE 6: The dynamics between fuzzy credit spreads and external shocks.

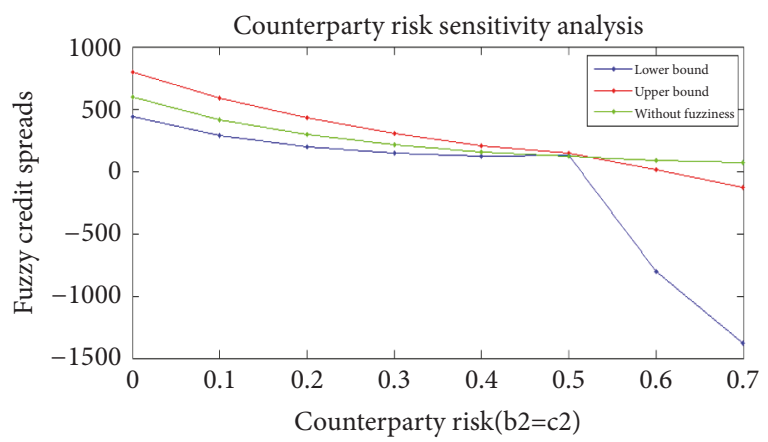

FIGURE 7: The dynamics between fuzzy credit spreads and counterparty risk.

\section{Conflicts of Interest}

The authors declare that there are no conflicts of interest regarding the publication of this paper. The authors confirm that the received funding, grants, or scholarships do not lead 
to any conflicts of interest regarding the publication of the manuscript.

\section{Authors' Contributions}

Liang $\mathrm{Wu}$ is the main writer of the framework and content of this article, Xian-bin Mei carried out numerical simulation, and Jian-guo Sun reviewed the full text.

\section{Acknowledgments}

The work was supported by the Project funded by China Postdoctoral Science Foundation (no. 2017M622325).

\section{References}

[1] D. Lando, "On cox processes and credit risky securities," Review of Derivatives Research, vol. 2, no. 2-3, pp. 99-120, 1998.

[2] D. Duffie and K. J. Singleton, "Modeling term structures of defaultable bonds," Review of Financial Studies , vol. 12, no. 4, pp. 687-720, 1999.

[3] R. A. Jarrow and F. Yu, "Counterparty risk and the pricing of defaultable securities," Journal of Finance, vol. 56, no. 5, pp. 1765-1799, 2001.

[4] S. Y. Leung and Y. K. Kwork, "Credit default swap valuation with counterparty risk," The Kyoto Economic Review, vol. 74, no. 1, pp. 25-45, 2005.

[5] Y.-f. Bai, X.-h. Hu, and Z.-x. Ye, "A model for dependent default with hypberbolic attenuation effect and valuation of credit default swap," Applied Mathematics and Mechanics-English Edition, vol. 28, no. 12, pp. 1643-1649, 2007.

[6] W. Li and L. Han, “The Fuzzy Binomial Option Pricing Model under Knightian Uncertainty," in Proceedings of the 2009 Sixth International Conference on Fuzzy Systems and Knowledge Discovery, pp. 399-403, Tianjin, China, August 2009.

[7] Y. Yoshida, "The valuation of European options in uncertain environment," European Journal of Operational Research, vol. 145, no. 1, pp. 221-229, 2003.

[8] H. Wu, "Using fuzzy sets theory and Black-Scholes formula to generate pricing boundaries of European options," Applied Mathematics and Computation, vol. 185, no. 1, pp. 136-146, 2007.

[9] W. Xu, C. Wu, W. Xu, and H. Li, "A jump-diffusion model for option pricing under fuzzy environments," Insurance: Mathematics \& Economics, vol. 44, no. 3, pp. 337-344, 2009.

[10] Y. Yoshida, M. Yasuda, J.-I. Nakagami, and M. Kurano, "A new evaluation of mean value for fuzzy numbers and its application to American put option under uncertainty," Fuzzy Sets and Systems, vol. 157, no. 19, pp. 2614-2626, 2006.

[11] H.-C. Wu, "European option pricing under fuzzy environments," International Journal of Intelligent Systems, vol. 20, no. 1, pp. 89-102, 2005.

[12] M. R. Hassan, K. Ramamohanarao, J. Kamruzzaman, M. Rahman, and M. Maruf Hossain, "A HMM-based adaptive fuzzy inference system for stock market forecasting," Neurocomputing, vol. 104, pp. 10-25, 2013.

[13] B. Q. Sun, H. F. Guo, H. R. Karimi, Y. Ge, and S. Xiong, "Prediction of stock index futures prices based on fuzzy sets and multivariate fuzzy time series," Neurocomputing, vol. 151, no. 3, pp. 1528-1536, 2015.
[14] E. Agliardi and R. Agliardi, "Fuzzy defaultable bonds," Fuzzy Sets and Systems, vol. 160, no. 18, pp. 2597-2607, 2009.

[15] E. Agliardi and R. Agliardi, "Bond pricing under imprecise information," Operational Research, vol. 11, no. 3, pp. 299-309, 2011.

[16] P. G. Vassiliou, "Fuzzy semi-Markov migration process in credit risk," Fuzzy Sets and Systems, vol. 223, pp. 39-58, 2013.

[17] Y. F. Bai, Modelling Contagion Effect of Credit Risk and Pricing of [Ph.D. thesis], Shanghai Jiao Tong University, Shanghai, China, 2008.

[18] K. S. Leung and Y. K. Kwok, "Counterparty risk for credit default swaps: Markov chain interacting intensities model with stochastic intensity," Asia-Pacific Financial Markets, vol. 16, no. 3, pp. 169-181, 2009.

[19] L. Wu and Y. M. Zhuang, "A reduced-form intensity-based model under fuzzy environments," Communications in Nonlinear Science and Numerical Simulation, vol. 22, no. 1-3, pp. 11691177, 2015.

[20] L. Wu, Y. M. Zhuang, and W. Li, "A new default intensity model with fuzziness and hesitation," International Journal of Computational Intelligence Systems, vol. 9, no. 2, pp. 340-350, 2016.

[21] Xiandong Wang, Jianmin He, and Shouwei Li, "Compound Option Pricing under Fuzzy Environment," Journal of Applied Mathematics, vol. 2014, Article ID 875319, 9 pages, 2014.

[22] L. Wu, J.-T. Wang, J.-F. Liu, and Y.-M. Zhuang, "The Total Return Swap Pricing Model under Fuzzy Random Environments," Discrete Dynamics in Nature and Society, vol. 2017, 2017.

[23] Liang Wu, Yaming Zhuang, and Xiaojing Lin, "Credit Derivatives Pricing Model for Fuzzy Financial Market," Mathematical Problems in Engineering, vol. 2015, Article ID 879185, 6 pages, 2015.

[24] L. A. Zadeh, "Fuzzy sets," Information and Computation, vol. 8, pp. 338-353, 1965.

[25] A. Kaufmann and M. M. Gupta, Introduction to fuzzy arithmetic: Theory and applications, Von Nostrand Reinhold Company, New yrok, NY, USA, 1985.

[26] H.-C. Wu, "Pricing European options based on the fuzzy pattern of Black-Scholes formula," Computers \& Operations Research, vol. 31, no. 7, pp. 1069-1081, 2004.

[27] M. L. Puri and D. A. Ralescu, "Fuzzy random variables," Journal of Mathematical Analysis and Applications, vol. 114, no. 2, pp. 409-422, 1986.

[28] P. Collin-Dufresne, R. Goldstein, and J. Hugonnier, "A general formula for valuing defaultable securities," Econometrica, vol. 72, no. 5, pp. 1377-1407, 2004.

[29] S. E. Shreve, Stochastic Calculus for Finance I: The Binomial Asset Pricing Model, Springer, New York, NY, USA, 2004.

[30] R. L. Hao, Pricing Credit Securities in the Contagious Model [Ph.D. thesis], Shanghai Jiao Tong University, Shanghai, China, 2011. 


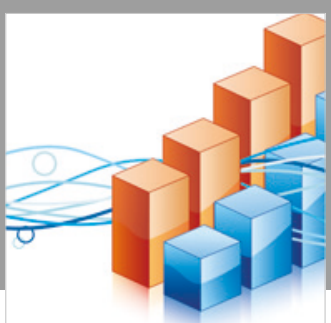

Advances in

Operations Research

\section{-n-m}
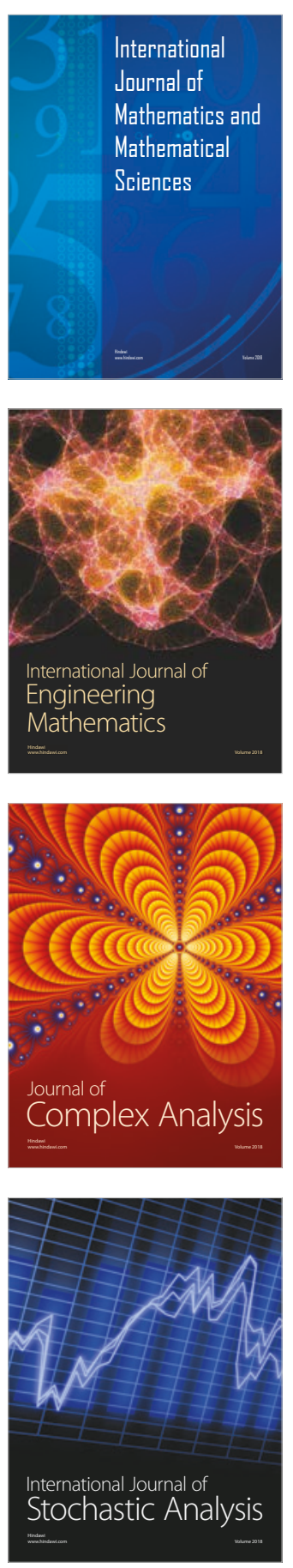
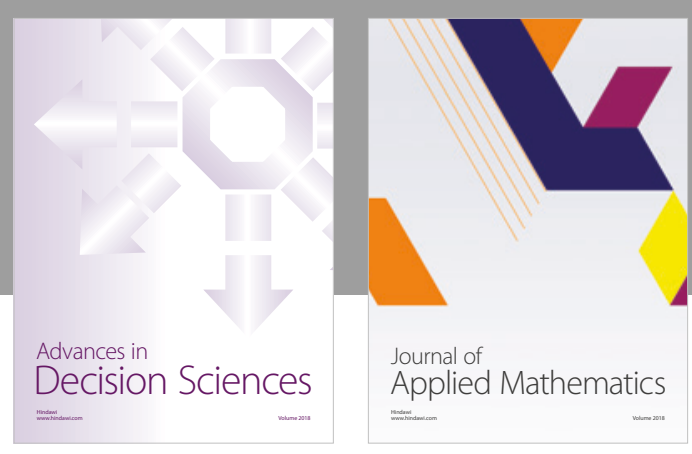

Journal of

Applied Mathematics
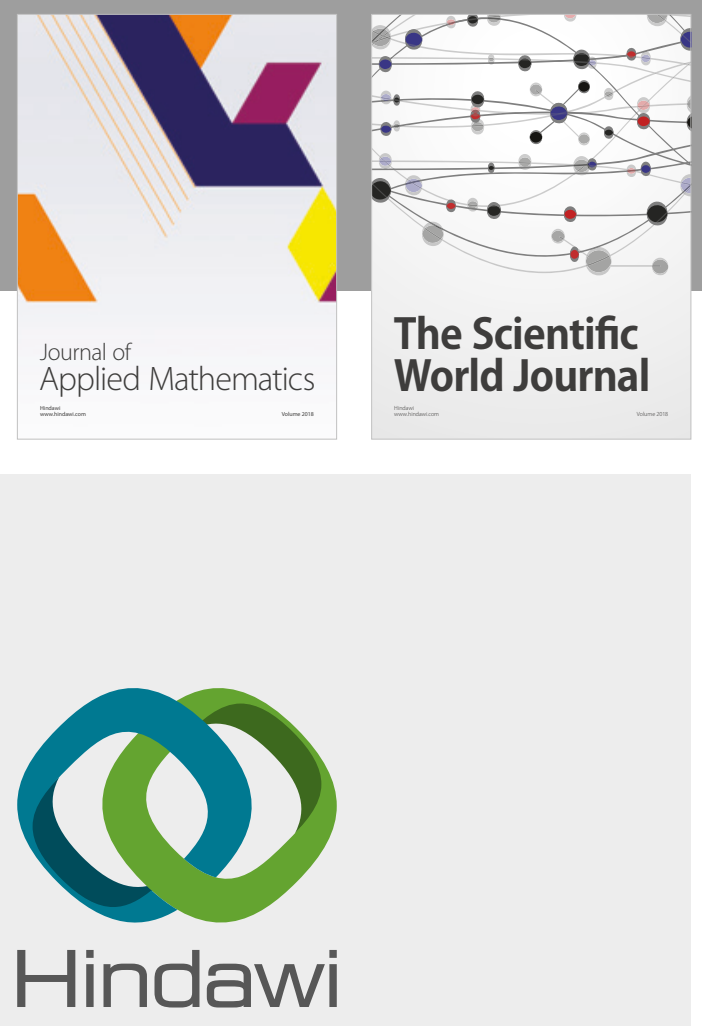

Submit your manuscripts at

www.hindawi.com

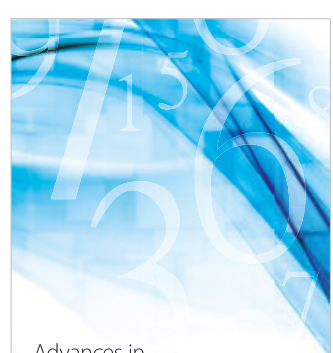

Advances in
Numerical Analysis
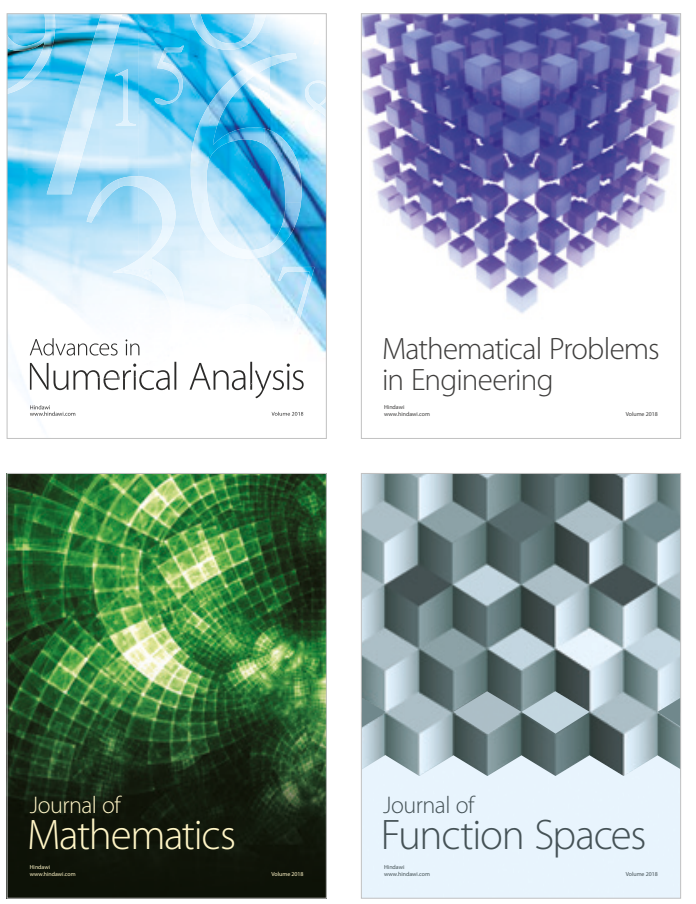

Mathematical Problems in Engineering

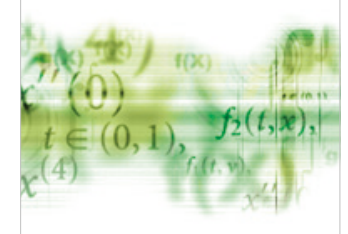

International Journal of

Differential Equations

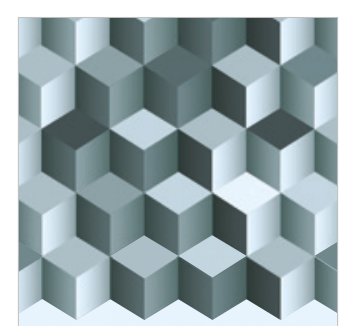

Journal of

Function Spaces

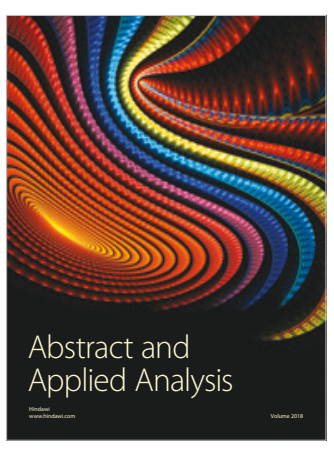

The Scientific

World Journal

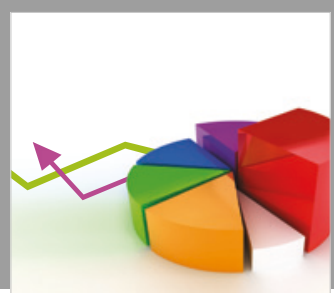

Journal of

Probability and Statistics
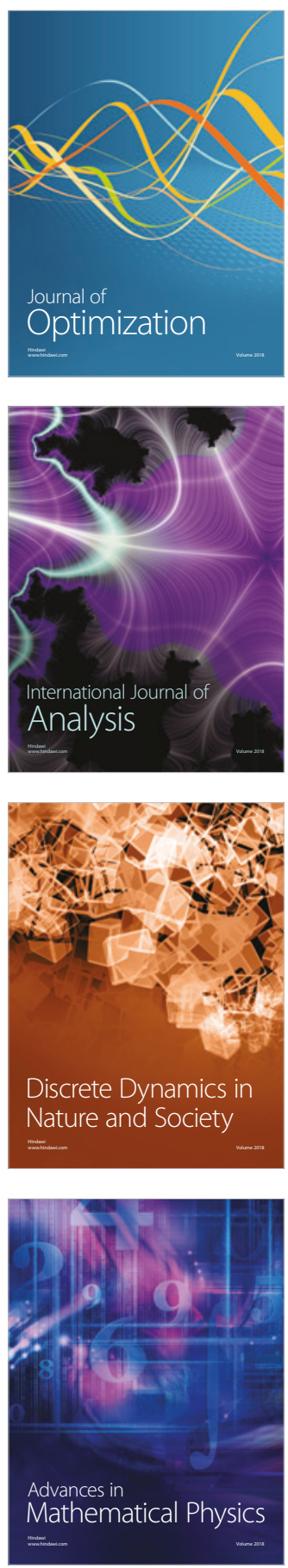